\title{
Risk Factors of In-Hospital Lethal Arrhythmia Following Acute Myocardial Infarction in Patients Undergoing Primary Percutaneous Coronary Intervention
}

\author{
- Insight From the J-MINUET Study -
}

\author{
Jun Oikawa, MD, PhD; Hidehira Fukaya, MD, PhD; Junya Ako, MD, PhD; \\ Koichi Nakao, MD, PhD; Yukio Ozaki, MD, PhD; Kazuo Kimura, MD, PhD; \\ Teruo Noguchi, MD, PhD; Satoru Suwa, MD, PhD; Kazuteru Fujimoto, MD, PhD; \\ Yasuharu Nakama, MD; Takashi Morita, MD, PhD; Wataru Shimizu, MD, PhD; \\ Yoshihiko Saito, MD, PhD; Atsushi Hirohata, MD, PhD; Yasuhiro Morita, MD, PhD; \\ Teruo Inoue, MD, PhD; Atsunori Okamura, MD, PhD; Toshiaki Mano, MD, PhD; \\ Yoshihiro Miyamoto, MD, PhD; Hisao Ogawa, MD, PhD; Masaharu Ishihara, MD, PhD \\ on behalf the J-MINUET Investigators
}

\begin{abstract}
Background: Lethal arrhythmias including ventricular tachycardia and fibrillation (VT/VF) are common complications of acute myocardial infarction (AMI). Predictors of in-hospital VT/VF after AMI, however, have not been thoroughly investigated. In this study, we sought to elucidate the predictors of in-hospital VT/VF events after AMI in the Japanese registry of acute Myocardial INfarction diagnosed by Universal dEfiniTion (J-MINUET).

Methods and Results: In-hospital VT/VF was defined as a hemodynamically unstable VT or VF in the first week of hospitalization, on which the patients were classified as the VT/VF group. Of the patients in the J-MINUET study, 3,175 were finally enrolled in this study. A total of 114 patients had VT/VF. On multivariate logistic analysis, maximum creatine kinase >3,000 IU/L (adjusted OR, 1.67; 95\% Cl: 1.085-2.572; $\mathrm{P}=0.02$ ), Killip class III or IV (adjusted OR, 8.93; 95\% Cl: 5.668-14.082; $\mathrm{P}<0.0001$ ), initial Thrombolysis in Myocardial Infarction (TIMI) flow grade 0 or 1 (adjusted OR, 1.67; 95\% Cl: 1.035-2.709; P=0.03), and concomitant chronic kidney disease (CKD; adjusted OR, 1.80; 95\% Cl: 1.105-2.938; P=0.02) were identified as independent predictors for in-hospital VT/VF.
\end{abstract}

Conclusions: From the J-MINUET study, extensive myocardial damage, cardiogenic shock, lower grade initial TIMI flow on coronary angiography, and concomitant CKD were independent predictors of in-hospital VT/VF after AMI.

Key Words: Acute myocardial infarction; J-MINUET study; Ventricular fibrillation; Ventricular tachycardia

A cute myocardial infarction (AMI) is still a lifethreating disease..$^{\mathbf{1}, 2}$ Although primary percutaneous coronary intervention (PCI) is now carried out as first-line therapy, arrhythmias including ventricular tachycardia and fibrillation (VT/VF) after AMI are still lethal major complications even after successful primary PCI. ${ }^{3}$
Predictive factors for lethal arrhythmia after primary PCI for AMI, however, remain undetermined.

In this study, we elucidated the predictors of in-hospital VT/VF events following during hospitalization for primary PCI using the Japanese registry of acute Myocardial INfarction diagnosed by Universal dEfiniTion (J-MINUET),

Received September 20, 2019; revised manuscript received November 28, 2019; accepted December 3, 2019; J-STAGE Advance Publication released online December 27, 2019 Time for primary review: 1 day

Department of Cardiovascular Medicine, Kitasato University School of Medicine, Sagamihara (J.O., H.F., J.A.); Cardiovascular Center, Saiseikai Kumamoto Hospital, Kumamoto (K.N.); Department of Cardiovascular Medicine, Fujita Health University, Toyoake (Y.O.); Department of Cardiology, Yokohama City University Medical Center, Yokohama (K.K.); Department of Cardiovascular Medicine (T.N., H.O.), Department of Preventive Cardiology (Y. Miyamoto), National Cerebral and Cardiovascular Center, Suita; Department of Cardiology, Juntendo University Shizuoka Hospital, Izunokuni (S.S.); Department of Cardiology, National Hospital Organization Kumamaoto Medical Center, Kumamoto (K.F.); Department of Cardiology, Hiroshima City Hiroshima Citizens Hospital, Hiroshima (Y.N.); Department of Cardiology, Osaka General Medical Center, Osaka (T. Morita); Department of Cardiovascular Medicine, Nippon Medical School, Tokyo (W.S.); Department of Cardiovascular Medicine, Nara Medical University, Kashihara (Y.S.); Department of Cardiovascular Medicine, The Sakakibara Heart Institute of Okayama, Okayama (A.H.); Department of Cardiology, Ogaki Municipal Hospital, Ogaki (Y. Morita); 


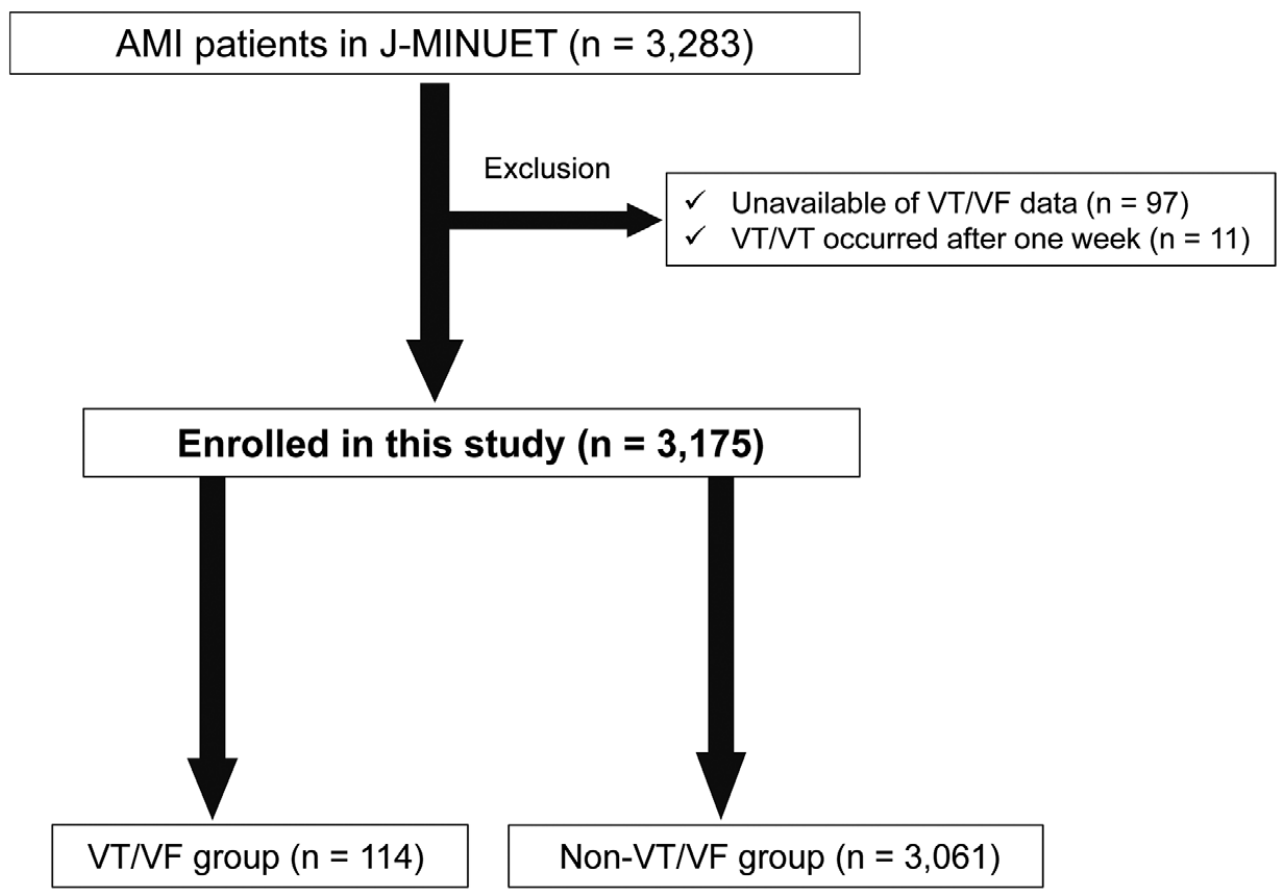

Figure. Subject selection. AMI, acute myocardial infarction; J-MINUET, Japanese registry of acute Myocardial INfarction diagnosed by Universal dEfiniTion; VF, ventricular fibrillation; VT, ventricular tachycardia.

a multicenter registry of Japanese patients with AMI. ${ }^{4}$

\section{Methods}

\section{Subjects}

The J-MINUET study is a prospective, observational multicenter registry of Japanese patients hospitalized for AMI as diagnosed by the universal definition (UMIN000010037). ${ }^{4}$ A total of 3,283 consecutive patients who were admitted $\leq 48 \mathrm{~h}$ after onset of AMI at 28 Japanese medical institutions during July 2012 through March 2014 were enrolled in that study. AMI diagnosis was based on the ESC/ACCF/AHA/ World Heart Federation Task Force for the Universal Definition of Myocardial Infarction..$^{5}$ This registry included only type 1 (spontaneous myocardial infarction $[\mathrm{MI}]$ related to ischemia from a primary coronary event) and type 2 [MI secondary to ischemia due to either increased oxygen demand or decreased oxygen supply). In addition, AMI was diagnosed as an increase in cardiac biomarkers (preferred: troponin) with at least 1 value above the 99th percentile of the upper reference limit, together with the evidence of myocardial ischemia with at least one of the following: symptoms of ischemia, electrocardiogram changes indicative of new ischemia or development of pathological Q waves, and imaging evidence of a new loss of viable myocardium or new regional wall motion abnormalities.

All patients were divided into 2 groups based on the incidence of lethal arrhythmia in the acute phase: the VT/ $\mathrm{VF}$ or the non-VT/VF groups. Lethal arrhythmia in the acute phase was defined as sustained VT or VF requiring defibrillation or cardiopulmonary resuscitation $\leq 1$ week after the onset of AMI during hospitalization, consistent with a previous study. ${ }^{6}$ To elucidate the predictors of inhospital VT/VF, the patient characteristics, comorbidities, clinical manifestations, and coronary angiography were compared between the 2 groups. Most laboratory data were measured at admission. Frequency and time interval of creatine kinase $(\mathrm{CK})$ measurement were not prespecified but left to the physician's decision. This study was conducted in accordance with the Declaration of Helsinki and this protocol was approved by the ethics committees of every participating institution.

\section{Statistical Analysis}

Statistical analysis was performed using JMP 13 (SAS Institute, Cary, NC, USA). Continuous variables were compared using the Mann-Whitney U-test when applicable. Data are presented as median (IQR). Dichotomous variables are presented as percentages and were compared using the chi-squared test. Multivariate logistic regression

Department of Cardiovascular Medicine, Dokkyo Medical University, Tochigi (T.I.); Department of Cardiology, Sakurabashi Watanabe Hospital, Osaka (A.O.); Cardiovascular Center, Kansai Rosai Hospital, Amagasaki (T. Mano); and Division of Cardiovascular and Renal Medicine, Hyogo College of Medicine, Nishinomiya (M.I.), Japan

J.A., Y.O., T.I., and Y. Miyamoto are members of Circulation Reports' Editorial Team.

Mailing address: Jun Oikawa, MD, PhD, Department of Cardiovascular Medicine, Kitasato University School of Medicine, 1-15-1

Kitasato, Minami-ku, Sagamihara 252-0374, Japan. E-mail: joichan@med.kitasato-u.ac.jp

ISSN-2434-0790 All rights are reserved to the Japanese Circulation Society. For permissions, please e-mail: cr@j-circ.or.jp 


\begin{tabular}{|c|c|c|c|}
\hline Age (years) & $69(61-78)$ & Angiography & \\
\hline Male & $2,391(75)$ & Culprit vessel & \\
\hline \multicolumn{2}{|l|}{ Presenting characteristics } & Left main & $53(2)$ \\
\hline $\mathrm{HR}$ (beats/min) & $77(65-90)$ & Left anterior descending & $1,132(36)$ \\
\hline $\mathrm{SBP}(\mathrm{mmHg})$ & $139(118-160)$ & Left circumflex & $352(11)$ \\
\hline LVEF (\%) & $50(40-59)$ & Right coronary & $997(31)$ \\
\hline STEMI & $2,184(69)$ & Multi-vessel & $1,289(44)$ \\
\hline Preconditioning & $1,043(38)$ & Initial TIMI flow & \\
\hline Onset to admission $<6 \mathrm{~h}$ & $2,338(74)$ & Grade 0 & $1,462(50)$ \\
\hline DTB time $<90 \mathrm{~min}$ & $1,533(64)$ & Grade I & $311(10)$ \\
\hline LDL-C/HDL-C ratio & $2.5(1.9-3.3)$ & Grade II & $496(17)$ \\
\hline Blood sugar (mg/dL) & $150(121-150)$ & Grade III & $659(23)$ \\
\hline Serum potassium (mEq/L) & $4.0(3.7-4.4)$ & Underlying disease & \\
\hline eGFR $\left(\mathrm{mL} / \mathrm{min} / 1.73 \mathrm{~m}^{2}\right)$ & $64.7(48.1-81.2)$ & IHD & $531(17)$ \\
\hline Maximum CK (IU/L) & $1,431(502-3,149)$ & CKD (grades 3-5) & $1,419(45)$ \\
\hline Killip class & & Hypertension & $2,098(67)$ \\
\hline 1 & $2,379(76)$ & Diabetes mellitus & $1,129(36)$ \\
\hline II & $296(9)$ & Dyslipidemia & $1,633(52)$ \\
\hline III & $172(5)$ & Prescription & \\
\hline \multirow[t]{4}{*}{ IV } & $308(10)$ & ARB/ACEI & $990(31)$ \\
\hline & & $\beta$-blockers & 453 (14) \\
\hline & & $\mathrm{CCB}$ & 1,088 (34) \\
\hline & & Statins & 735 (23) \\
\hline
\end{tabular}

Data given as $\mathrm{n}(\%)$ or median (IQR). ACEI, angiotensin-converting enzyme inhibitors; ARB, angiotensin II receptor blockers; CCB, calcium-channel blockers; CK, creatine kinase; CKD, chronic kidney disease; DTB, door to balloon; eGFR, estimated glomerular filtration rate; HDL-C, high-density lipoprotein cholesterol; HR, heart rate; IHD, ischemic heart disease; LDL-C, low-density lipoprotein cholesterol; LVEF, left ventricular ejection fraction; SBP, systolic blood pressure; STEMI, ST-segment elevation myocardial infarction; TIMI, Thrombolysis in Myocardial Infarction.

analysis with a stepwise method, using parameters that were significantly different on univariate analysis, was used to identify independent factors to predict in-hospital VT/VF, while some factors with potential confounding were excluded. $\mathrm{P}<0.05$ was considered to be statistically significant.

\section{Results}

\section{Patient Characteristics}

A total of 3,283 patients were assigned in the J-MINUET study. One hundred and eight patients were excluded because VT/VF data were unavailable $(n=97)$ or lethal arrhythmia occurred after 1 week $(n=11)$, after which 3,175 patients were finally enrolled in this study (Figure). The median age was 69 years, and $25 \%$ were female. Table 1 lists patient clinical background and the characteristics of AMI in this study. In-hospital VT/VF events were observed in 114 patients (VT/VF group), which was $3.7 \%$ of the total enrolled patients (the others were defined as the non-VT/ VF group; $n=3,061)$.

\section{Patient Characteristics vs. VT/NF Status}

Table 2 lists the patient characteristics and the details of clinical manifestation on admission in both groups. The VT/VF group had higher heart rate $(\mathrm{HR} ; \mathrm{P}<0.0001)$, and lower systolic blood pressure (SBP) compared with the non-VT/VF group. The VT/VF group had fewer prior chest pain symptoms, and shorter transfer time from onset to admission compared with the non-VT/VF group. The patients in the VT/VF group had a higher prevalence of
ST-segment elevation MI (STEMI), and a higher severity Killip classification compared with the non-VT/VF group, which also suggests that the VT/VF group had a more severe condition on admission.

With regard to laboratory data, the VT/VF group also had higher blood sugar on admission, lower estimated glomerular filtration rate (eGFR), and a higher proportion of maximum CK compared with the non-VT/VF group. Table 3 lists the comorbidities and prior medication for the 2 groups. The VT/VF group had a higher prevalence of comorbidity of chronic kidney disease (CKD; grade 3-5). There were no significant differences in any of the prior medications between the 2 groups. Table 4 summarizes the angiography data. The VT/VF group had a higher prevalence of left main trunk stenosis, multi-vessel coronary stenosis, and initial Thrombolysis in Myocardial Infarction (TIMI) flow grade 0 or 1 compared with the non-VT/VF group, suggesting that the VT/VF group had more severe or more complex lesions.

\section{Multivariate Analysis}

Table 5 lists the results of the multivariate logistic analysis with stepwise analysis of clinical parameters that were significantly different on univariate analysis. HR, SBP, and eGFR, however, were excluded from the multivariate analysis because these factors may have multiple collinearity or be clinically related. Consequently, a shorter time from the onset of symptom to arrival $<6 \mathrm{~h}$, maximum $\mathrm{CK}$ $>3,000$ IU/L, higher severity Killip classification on admission, initial TIMI flow grade 0 or 1 , and concomitant CKD were identified as independent factors for VT/VF. A total 


\begin{tabular}{|c|c|c|c|}
\hline & $\begin{array}{c}\text { VT/VF group } \\
(n=114)\end{array}$ & $\begin{array}{c}\text { Non-VT/VF group } \\
(n=3,061)\end{array}$ & P-value \\
\hline Age & $70(60-79)$ & $69(61-78)$ & 0.94 \\
\hline Male & $89(78)$ & $2,301(75)$ & 0.48 \\
\hline \multicolumn{4}{|l|}{ Presenting characteristics } \\
\hline HR (beats/min) & $97(73-111)$ & $77(65-90)$ & $<0.0001^{*}$ \\
\hline $\mathrm{SBP}(\mathrm{mmHg})$ & $118(94-156)$ & $139(119-160)$ & $<0.0001^{*}$ \\
\hline LVEF (\%) & $48.2(40.3-57.0)$ & $50.0(40.0-59.0)$ & 0.45 \\
\hline STEMI & $93(82)$ & $2,093(68)$ & $0.0028^{*}$ \\
\hline Preconditioning & $28(25)$ & $1,014(33)$ & $0.03^{*}$ \\
\hline Onset to admission $<6 \mathrm{~h}$ & $102(89)$ & $2,235(73)$ & $<0.0001^{*}$ \\
\hline Door to balloon time $<90 \mathrm{~min}$ & $62(54)$ & $1,470(48)$ & 0.07 \\
\hline LDL-C/HDL-C ratio & $2.4(1.9-3.2)$ & $2.5(1.9-3.3)$ & 0.27 \\
\hline Blood sugar (mg/dL) & $206(145-287)$ & $149(120-200)$ & $<0.0001^{*}$ \\
\hline Serum potassium (mEq/L) & $4.1(3.6-4.6)$ & $4.0(3.7-4.4)$ & 0.48 \\
\hline eGFR $\left(\mathrm{mL} / \mathrm{min} / 1.73 \mathrm{~m}^{2}\right)$ & $49.69(38.10-65.17)$ & $65.39(48.60-81.63)$ & $<0.0001^{*}$ \\
\hline Maximum CK (IU/L) & $3,293(1,404-7,585)$ & $1,392(493-3,070)$ & $<0.0001^{*}$ \\
\hline Maximum $\mathrm{CK}>3,000 \mathrm{IU} / \mathrm{L}$ & $60(53)$ & $795(26)$ & $<0.0001^{*}$ \\
\hline Killip class III or IV & $74(65)$ & $401(13)$ & $<0.0001^{*}$ \\
\hline
\end{tabular}

Data given as $\mathrm{n}(\%)$ or median (IQR). ${ }^{*} \mathrm{P}<0.05$. AMI, acute myocardial infarction; VF, ventricular fibrillation; $\mathrm{VT}$, ventricular tachycardia. Other abbreviations as in Table 1.

\begin{tabular}{|c|c|c|c|}
\hline & $\begin{array}{c}\text { VT/VF group } \\
(n=114)\end{array}$ & $\begin{array}{c}\text { Non-VT/VF group } \\
(n=3,061)\end{array}$ & P-value \\
\hline \multicolumn{4}{|l|}{ Underlying disease } \\
\hline Ischemic heart disease & $22(19)$ & $510(17)$ & 0.46 \\
\hline CKD (grade 3-5) & $82(72)$ & $1,335(44)$ & $<0.0001^{*}$ \\
\hline Hypertension & $69(61)$ & $2,033(66)$ & 0.25 \\
\hline Diabetes mellitus & $41(36)$ & $1,084(35)$ & 0.86 \\
\hline Dyslipidemia & $51(45)$ & $1,585(52)$ & 0.22 \\
\hline \multicolumn{4}{|l|}{ Prior medications } \\
\hline ARB/ACEI & $37(32)$ & 956 (31) & 0.78 \\
\hline$\beta$-blockers & $18(16)$ & $434(14)$ & 0.63 \\
\hline CCB & $34(30)$ & $1,056(34)$ & 0.30 \\
\hline Statins & $30(26)$ & 704 (23) & 0.43 \\
\hline
\end{tabular}

Data given as $n(\%) .{ }^{*} \mathrm{P}<0.05$. Abbreviations as in Tables 1,2 .

\begin{tabular}{|c|c|c|c|}
\hline & $\begin{array}{l}\text { VT/VF group } \\
(n=114)\end{array}$ & $\begin{array}{c}\text { Non-VT/VF group } \\
(n=3,061)\end{array}$ & P-value \\
\hline \multicolumn{4}{|l|}{ Angiography parameters } \\
\hline \multicolumn{4}{|l|}{ Culprit vessel } \\
\hline Left main & $11(10)$ & $42(1)$ & $<0.0001^{*}$ \\
\hline Left anterior descending & $36(32)$ & $1,093(36)$ & 0.37 \\
\hline Left circumflex & $9(8)$ & $343(11)$ & 0.27 \\
\hline Right coronary & $35(31)$ & $943(31)$ & 0.98 \\
\hline Multi-vessel & $62(54)$ & $1,224(40)$ & $0.0052^{*}$ \\
\hline \multicolumn{4}{|l|}{ Initial TIMI flow } \\
\hline Grade 0 or I & $82(72)$ & $1,687(55)$ & $0.0008^{*}$ \\
\hline \multicolumn{4}{|l|}{ Final TIMI flow } \\
\hline Grade 0 or I & $6(5)$ & $76(2)$ & 0.14 \\
\hline
\end{tabular}

Data given as $n(\%)$. ${ }^{*}<0.05$. Abbreviations as in Tables 1,2 . 


\begin{tabular}{|c|c|c|c|}
\hline & Wald Chi-squared & OR (95\% Cl) & P-value \\
\hline Age & 1.32 & $0.98(0.972-1.008)$ & 0.25 \\
\hline Gender & 0.60 & $1.23(0.732-2.052)$ & 0.44 \\
\hline Onset to admission $<6 \mathrm{~h}$ & 4.97 & $2.05(1.091-3.837)$ & $0.03^{*}$ \\
\hline Maximum CK >3,000 IU/L & 5.43 & 1.67 (1.085-2.572) & $0.02^{*}$ \\
\hline Killip class III or IV & 88.95 & $8.93(5.668-14.082)$ & $<0.0001^{*}$ \\
\hline Initial TIMI flow 0 or 1 & 4.41 & 1.67 (1.035-2.709) & $0.03^{*}$ \\
\hline CKD (grade 3-5) & 5.57 & $1.80(1.105-2.938)$ & $0.02^{*}$ \\
\hline
\end{tabular}

${ }^{*} \mathrm{P}<0.05$. Abbreviations as in Tables 1,2 .

of 123 of the present patients $(4 \%)$ had all 4 risk factors.

\section{Discussion}

In this multicenter Japanese registry, we identified the following factors to predict in-hospital VT/VF in the acute phase on univariate analysis: faster HR, decreased SBP, lower prevalence of preceding symptom of chest pain, shorter time from onset to admission, STEMI, multi-vessel coronary stenosis, initial TIMI flow grade 0 or 1 , concomitant CKD, hyperglycemia on admission, and higher maximum CK. On multivariate analysis, shorter time from onset to hospital arrival $<6 \mathrm{~h}$, maximum $\mathrm{CK}>3,000 \mathrm{IU} / \mathrm{L}$, higher severity Killip classification on admission, initial TIMI flow grade 0 or 1 , and concomitant CKD were independent risk factors for in-hospital VT/VF in the AMI patients undergoing primary PCI in Japan.

\section{Incidence and Predictors of Lethal Arrhythmia After AMI}

The incidence of these lethal arrhythmias has been decreasing since primary PCI became the standard therapy for AMI, ${ }^{7}$ but they remain a fatal complication. In the PAMI trial and the HORIZONS-AMI trial performed in the USA, the incidences of in-hospital VT/VF were $4.3 \%$ and $5.2 \%$, respectively, ${ }^{\mathbf{8} 9}$ which is relatively consistent with the present study $(3.6 \%)$. According to the predictive factors for inhospital VT/VF, these trials identified several independent factors: lack of pre-procedural $\beta$-blockers or angiotensinconverting enzyme inhibitors/angiotensin receptor blockers (ACEI/ARB) on admission, time from symptom onset to emergency room $<180 \mathrm{~min}$, initial TIMI flow grade 0 , and right coronary artery-related infarction, and Killip class $>$ I. ${ }^{8,9}$ Similar to these reports, shorter time from symptom onset to admission, lower grade of initial TIMI flow grade, and worse grade of Killip class were also independent risk factors for in-hospital VT/VF in the present study. In addition, higher CK and concomitant CKD were also extracted as independent risk factors in this study. Although the indicated risk factors were slightly different from these previous studies, presumably because of the difference in patient characteristics, it is reasonable that the factors associated with more severe hemodynamic condition or severe angiography findings predict in-hospital VT/VF in the acute phase after PCI. Also, the aforementioned papers were reported from Europe and the USA, and there are few reports from Japan. It is of importance to evaluate the predictors of events in the Japanese population. Furthermore, in the previous studies, the definition of arrhythmia events included non-fatal non-sustained VT. The present study, however, focused on lethal arrhythmia requiring intervention. And concomitant CKD was an independent predictor of in-hospital VT/VF in this study, which has not been reported previously.

\section{Mechanism of Occurrence of Lethal Arrhythmia in AMI}

Local ischemia causes local impairment of the sympathetic nerve fibers, resulting in non-uniform sympathetic denervation. ${ }^{10}$ Increased sympathetic tone after AMI and ischemia itself lead to shortened electrical refractory periods in injured myocardium. This spatially non-uniform electrical disturbance between injured and normal myocardium causes reentrant VT/VF. With regard to other mechanisms, catecholamine sensitivity is increased in the area surrounding the infarcted myocardium due to upregulation of the catecholamine receptors after ischemic damage. ${ }^{11}$ These sites are prone to an increased automaticity, which also causes the ventricular ectopic beats preceding VT/VF. Also, the injured myocardium has impaired calcium cycling at a cellular level, which causes delayed afterdepolarization followed by triggered activity. ${ }^{12}$ In addition to the aforementioned mechanisms, left ventricular volume overload has been suggested to increase the spatial electrical dispersion between the abnormal and normal myocardium. ${ }^{13,14}$ Furthermore, the stretch-activated channels triggered by the extensive myocardial injury also cause the triggered activity. ${ }^{14}$ The factors of maximum CK $>3,000 \mathrm{IU} / \mathrm{L}$ or higher severity Killip classification identified as the VT/VF predictors in this study. These factor are basically related with a low cardiac function on the extensive AMI. Therefore, it is reasonable that the patients with higher CK or higher severity Killip class tend to be prone to the lethal arrhythmia.

In the other J-MINUET substudy, Hashimoto et al reported that a lower serum eicosapentaenoic acid/arachidonic acid ratio is associated with a higher prevalence of lethal arrhythmia in the early phase of AMI. The theoretical anti-arrhythmia mechanism of $n-3$ polyunsaturated fatty acids provides electrical stabilization of the sodium and calcium ion channels in the sarcolemma, ${ }^{15}$ which may prevent arrhythmias arising in the ischemic zone. Therefore, not only direct injury or mechanical stress by the myocardial ischemia/infarction, but also the patient's intrinsic vulnerability to the lethal arrhythmia might be associated with the occurrence of VT/VT in the acute phase after AMI.

\section{Reperfusion Arrhythmia After Myocardial Hypoperfusion}

Patients with VT/VF have been reported to have a higher prevalence of TIMI grade 0 flow before PCI, whereas TIMI grade 3 is an independent predictor of better outcome. ${ }^{16} \mathrm{In}$ animal experiments, the severity of ischemia was associated with the prevalence of reperfusion arrhythmia. ${ }^{17}$ Although immediate reperfusion is crucial to rescue the ischemic 
penumbra of myocardium, it also promotes the rate of formation of free radicals. The amount of free radicals is correlated with the area under hypoperfusion. ${ }^{18,19}$ The present finding that the patients with severe ischemia (TIMI 0 or 1 ) were prone to VT/VF may be related to the reperfusion injury and increased free radicals after PCI.

\section{CKD and Lethal Arrhythmia}

Stack and Bloembergen found that coronary artery disease patients with CKD have a higher mortality rate than those without CKD. ${ }^{20}$ In the present study, concomitant CKD was identified as a predictor of in-hospital lethal arrhythmia in the AMI patients after PCI. CKD patients also have a higher incidence of lethal arrhythmia than those without CKD, irrespective of the presence of ischemic heart disease, and the occurrence rate of lethal arrhythmia increases as eGFR decreases. ${ }^{21}$ Several possible reasons for why AMI patients with CKD are prone to VT/VF incidence, are as follows: reduced myocardial calcium ion homeostasis, ${ }^{22}$ excessive sympathetic nerve activity, ${ }^{23}$ resting membrane potential, and repolarization process abnormalities due to hyperkalemia or hypokalemia at the cellular level..24,25 Although serum potassium was not significantly different between the present groups, other factors may have been related to the occurrence of VT/VF in the patients with CKD.

\section{Time From AMI Onset to Admission}

A paradoxical observation in the present study was the relationship between occurrence of lethal arrhythmia and a shorter period from the onset of AMI to arrival at hospital. In general, a shorter time from onset to revascularization in patients with AMI is a predictor of better outcome. ${ }^{26}$ Lethal arrhythmias occur immediately after the onset of AMI, which is the main cause of early-phase cardiopulmonary arrest. With the development of prehospital services, however, such as ambulances and nontransporting emergency medical services vehicles in Japan, an increasing number of patients are being transported to hospitals in a shorter period. Similar results were reported in the Japanese OACIS study: that is, patients with acutephase VT/VF after primary PCI had a shorter time from onset of symptom to hospital arrival $<6 \mathrm{~h} .{ }^{6}$ Therefore, the patients with a hemodynamically unstable or more severe condition, such as cardiopulmonary arrest, may be quickly transferred to PCI sites. In addition, the patients who died of sustained lethal arrhythmias during transport to hospital or in emergency rooms were excluded from the registry, which poses a selection bias in this present study.

\section{Study Limitations}

The present investigation had several limitations. First, this study was a sub-analysis and retrospective study with a limited number of patients, therefore, this research may be influenced by biases and confounding factors. Second, given that this study was a post-hoc analysis, it was unclear when the lethal arrhythmia occurred: that is, immediately after admission, before revascularization, after revascularization, or during reperfusion with PCI, although they did all occur $\leq 1$ week after the onset of AMI. Third, other ventricular events such as premature ventricular contraction or non-fatal non-sustained VT, which might be related to future lethal arrhythmia, could not be evaluated because of a lack of these data in this retrospective study. Although $\mathrm{VT} / \mathrm{VF}$ during revascularization is more common, ${ }^{27}$ it is important that the risk in the early phase of hospitalization should be clarified. Further prospective studies are necessary to evaluate the predictors in detail.

\section{Conclusions}

In the J-MINUET registry, extensive myocardial damage, worse Killip classification, lower grade initial TIMI flow and concomitant CKD were independent predictors of inhospital lethal arrhythmia in patients with AMI after PCI.

\section{Acknowledgments}

This study was supported by an Intramural Research Fund, grant number 23-4-5, for Cardiovascular Diseases of the National Cerebral and Cardiovascular Center. The authors thank all the enrolled patients, participating cardiologists, medical and other staff who contributed to this study. The J-MINUET Investigators are listed in the Appendix.

\section{Disclosures}

The authors declare no conflicts of interest. J.A., Y.O., T.I., and Y. Miyamoto are members of Circulation Reports' Editorial Team.

\section{References}

1. Van der Wal AC, Becker AE, van der Loos CM. Site of intimal rupture or erosion of thrombosed coronary atherosclerotic plaques is characterized by an inflammatory process irrespective of the dominant plaque morphology. Circulation 1994; 89: $36-44$.

2. Kasanuki H, Honda T, Haze K, Sumiyoshi T, Horie T, Ogawa $\mathrm{H}$, et al; HIJAMI Investigators. A large-scale prospective cohort study on the current status of therapeutic modalities for acute myocardial infarction in Japan: Rationale and initial results of the HIJAMI Registry. Am Heart J 2005; 150: 411-418.

3. Behar S, Reicher-Reiss H, Shechter M, Rabinowitz B, Kaplinsky E, Kauli N, et al. Frequency and prognostic significance of secondary ventricular fibrillation complicating acute myocardial infarction: SPRINT Study Group. Am J Cardiol 1993; 7: $152-$ 156.

4. Ishihara M, Fujino M, Ogawa H, Yasuda S, Noguchi T, Miyamoto Y, et al. Clinical presentation, management and outcome of Japanese patients with acute myocardial infarction in the troponin era: Japanese Registry of Acute Myocardial Infarction Diagnosed by Universal Definition (J-MINUET). Circ J 2015; 79: 1255-1262.

5. Thygesen K, Alpert JS, Jaffe AS, Simoons ML, Chaitman BR, White HD, et al. Third universal definition of myocardial infarction. Circulation 2012; 126: 2020-2035.

6. Masuda M, Nakatani D, Hikoso S, Suna S, Usami M, Sakata Y, et al. Clinical impact of ventricular tachycardia and/or fibrillation during the acute phase of acute myocardial infarction on inhospital and 5-year mortality rates in the percutaneous coronary intervention era. Circ J 2016; 80: 1539-1547.

7. De Luca G, Suryapranata H, Ottervanger JP, Antman EM. Time delay to treatment and mortality in primary angioplasty for acute myocardial infarction: Every minute of delay counts. Circulation 2004; 109: 1223-1225.

8. Mehta RH, Harjai KJ, Boura J, Grines L, Stone GW, Grines $\mathrm{CL}$, et al. Sustained ventricular tachycardia or fibrillation in the cardiac catheterization laboratory among patients receiving primary percutaneous coronary intervention: Incidence, predictors, and outcomes. J Am Coll Cardiol 2004; 43: 1765-1772.

9. Mehta RH, Yu J, Piccini JP, Tcheng JE, Farkouh ME, Stone GW, et al. Prognostic significance of postprocedural sustained ventricular tachycardia or fibrillation in patients undergoing primary percutaneous coronary intervention (from the HORIZONS-AMI Trial). Am J Cardiol 2012; 109: 805-812.

10. Chen PS, Chen LS, Cao JM, Sharifi B, Karagueuzian HS, Fishbein MC. Sympathetic nerve sprouting, electrical remodeling and the mechanisms of sudden cardiac death. Cardiovasc Res 2001; 50: 409-416.

11. Dean JW, Lab MJ. Arrhythmia in heart failure: Role of mechanically induced changes in electrophysiology. Lancet 1989; 1: $1309-1312$

12. Pye MP, Cobbe SM. Arrhythmogenesis in experimental models 
of heart failure: The role of increased load. Cardiovasc Res 1996; 32: $248-257$

13. Iacoviello M, Forleo C, Guida P, Romito R, Sorgente A, Pitzalis M, et al. Ventricular repolarization dynamicity provides independent prognostic information toward major arrhythmic events in patients with idiopathic dilated cardiomyopathy. $\mathrm{J} \mathrm{Am} \mathrm{Coll}$ Cardiol 2007; 50: 225-231.

14. Fu L, Cao JX, Xie RS, Li J, Han Y, Dai YN, et al. The effect of streptomycin on stretch-induced electrophysiological changes of isolated acute myocardial infarcted hearts in rats. Europace 2007; 9: $578-584$.

15. Hashimoto T, Ako J, Nakao K, Ozaki Y, Kimura K, Ishihara $\mathrm{M}$, et al. A lower eicosapentaenoic acid/arachidonic acid ratio is associated with in-hospital fatal arrhythmic events in patients with acute myocardial infarction: A J-MINUET substudy. Heart Vessels 2018; 33: 481-488.

16. Brener SJ, Mehran R, Brodie BR, Guagliumi G, Witzenbichler B, Stone GW. Predictors and implications of coronary infarct artery patency at initial angiography in patients with acute myocardial infarction (from the CADILLAC and HORIZONS-AMI Trials). Am J Cardiol 2011; 108: 918-923.

17. Majidi M, Kosinski AS, Al-Khatib SM, Lemmert ME, Smolders L, Krucoff MW. Reperfusion ventricular arrhythmia 'bursts' predict larger infarct size despite TIMI 3 flow restoration with primary angioplasty for anterior ST-elevation myocardial infarction. Eur Heart J 2009; 30: 757-764.

18. Manning AS, Manning AS, Hearse DJ. Reperfusion-induced arrhythmias: Mechanisms and prevention. J Mol Cell Cardiol 1984; 16: 497-518.

19. Kaplinsky E, Ogawa S, Michelson EL, Dreifus LS. Instantaneous and delayed ventricular arrhythmias after reperfusion of acutely ischemic myocardium: Evidence for multiple mechanisms. Circulation 1981; 63: 333-340.

20. Stack AG, Bloembergen WE. Prevalence and clinical correlates of coronary artery disease among new dialysis patients in the United States: A cross-sectional study. J Am Soc Nephrol 2001; 12: $1516-1523$.

21. Pun PH, Smarz TR, Honeycutt EF, Shaw LK, Al-Khatib SM, Middleton JP. Chronic kidney disease is associated with increased risk of sudden cardiac death among patients with coronary artery disease. Kidney Int 2009; 76: 652-658.

22. Brosius FC 3rd, Hostetter TH, Kelepouris E, Mitsnefes MM, Moe SM, Wilson PW. Detection of chronic kidney disease in patients with or at increased risk of cardiovascular disease. Circulation 2006; 114: 1083-1087.

23. Pun PH. The interplay between CKD, sudden cardiac death, and ventricular arrhythmias. Adv Chronic Kidney Dis 2014; 21: 480-
488.

24. Bakris GL, Hart P, Ritz E. Beta blockers in the management of chronic kidney disease. Kidney Int 2006; 70: 1905-1913.

25. Pun PH, Lehrich RW, Honeycutt EF, Herzog CA, Middleton JP. Modifiable risk factors associated with sudden cardiac arrest within hemodialysis clinics. Kidney Int 2011; 79: 218-227.

26. O'Doherty M, Tayler DI, Quinn E, Vincent R, Chamberlain DA. Five hundred patients with myocardial infarction monitored within one hour of symptoms. Br Med J 1983; 286: 1405-1408.

27. Kuga H, Ogawa K, Oida A, Taguchi I, Nakatsugawa M, Hoshi $\mathrm{T}$, et al. Administration of atrial natriuretic peptide attenuates reperfusion phenomena and preserves left ventricular regional wall motion after direct coronary angioplasty for acute myocardial infarction. Circ J 2003; 67: 443-448.

\section{Appendix. J-MINUET Investigators}

Masaharu Ishihara, Hyogo College of Medicine; Ogawa Hisao, National Cerebral and Cardiovascular Center, Kumamoto University Graduate School of Medical Sciences; Nobuaki Kokubu, Sapporo Medical University; Tadaya Sato, Akita Medical Center; Teruo Inoue, Dokkyo Medical University; Shigeru Oshima, Gunma Prefectural Cardiovascular Center; Hiroshi Funayama, Saitama Medical Center Jichi Medical University; Ken Kozuma, Hiroyuki Kyono, Teikyo University; Wataru Shimizu, Nippon Medical School; Satoru Suwa, Juntendo University Shizuoka Hospital; Kengo Tanabe, Mitsui Memorial Hospital; Tetsuya Tobaru, Sakakibara Heart Institute; Kazuo Kimura, Yokohama City University Medical Center; Junya Ako, Kitasato University; Mafumi Owa, Suwa Red Cross Hospital; Yasuhiro Morita, Ogaki Municipal Hospital; Yukio Ozaki, Fujita Health University; Satoshi Yasuda, Teruo Noguchi, Masashi Fujino, National Cerebral and Cardiovascular Center; Junichi Kotani, Osaka University Graduate School of Medicine; Takashi Morita, Osaka General Medical Center; Atsunori Okamura, Sakurabashi Watanabe Hospital; Yoshihiko Saito, Nara Medical University; Masaaki Uematsu, Kansai Rosai Hospital; Hiroyuki Okura, Kawasaki Medical School; Atsushi Hirohata, The Sakakibara Heart Institute of Okayama; Yasuharu Nakama, Hiroshima City Hospital; Keijiro Saku, Fukuoka University School of Medicine; Seiji Hokimoto, Kumamoto University Graduate School of Medical Sciences; Koichi Nakao, Saiseikai Kumamoto Hospital; Kazuteru Fujimoto, National Hospital Organization Kumamoto Medical Center; Yoshisato Shibata, Miyazaki Medical Association Hospital; Kazuhito Hirata, Okinawa Prefectural Chubu Hospital; Yoshihiro Miyamoto, Kunihiko Nishimura, Michikazu Nakai, National Cerebral and Cardiovascular Center. 